\title{
Experimental study of air delivery into water- conveyance system of the radial-axial turbine
}

\author{
Alexandra Maslennikova ${ }^{1}$, Dmitry Platonov $^{1,2}{ }^{*}$, Andrey Minakov ${ }^{1,2}$, and Dmitry Dekterev ${ }^{2}$ \\ ${ }^{1}$ Siberean Federal University, 79 Svobodny pr., 660041 Krasnoyarsk, Russia \\ ${ }^{2}$ Institute of Thermophysics, SB RAS, 1 Lavrentyev pr., 630090, Novosibirsk, Russia
}

\begin{abstract}
The paper presents an experimental study of oscillatory response in the Francis turbine of hydraulic unit. The experiment was performed on large-scale hydrodynamic test-bench with impeller diameter of $0.3 \mathrm{~m}$. The effect of air injection on the intensity of pressure pulsations was studied at the maximum pressure pulsations in the hydraulic unit. It was revealed that air delivery into the water-conveyance system of the turbine results in almost two-fold reduction of pressure pulsations.
\end{abstract}

\section{Introduction}

Power control in the energy system is an important task of hydropower plant. When changing load of hydraulic unit, the latter repeatedly undergoes off-design modes of operation. In these modes, the flow remains essentially swirling after passing through the water turbine runner. The instability of swirling flow results in the appearance of intense low-frequency hydrodynamic pulsations, which threaten the safety and reliability of turbine design [1-4].

The main reason for the occurrence of low-frequency pressure pulsations is the precessing vortex core generated in the diffuser of the draft tube due to essential residual swirl of the flow.

Up to date, numerous methods are proposed to stabilize flow in the hydraulic units [58]. Each of them has its advantages and disadvantages, and requires further detailed study. In this study, the effect of air delivery on the oscillatory response of the flow was experimentally investigated on the model hydrodynamic Francis turbine test-bench.

\section{Experimental techniques}

The model hydroturbine with radial-axial impeller is part of the hydro-power test-bench at the Laboratory of Hydraulics and Hydraulic Machines of the Siberian Federal University (Fig. 1.). Maximum water head in the test-bench is $3.5 \mathrm{~m}$, the diameter of the impeller is $\mathrm{D}=0.3 \mathrm{~m}$.

* Corresponding author: platonov-08@yandex.ru 
Hydraulic unit includes a pressure tank, performing the functions of the upper pool, power conduit with the diameter of $400 \mathrm{~mm}$, and penstock valve, equipped with electric drive.

Water from the penstock enters the spiral chamber, where from flows through the guide vanes to the impeller and then passing the draft tube gets into the lower pool, which is a glass groove $1.52 \mathrm{~m}$ in width. Diffusion grid is designed to reduce fluctuations in water level before weir gauge. Weir gauge is used for measuring water flow rate and is fixed by water gauge.

After weir gauge the water is discharged into the collecting tank and then is diverted through pipe into the pool. From the pool, water is pumped into the pressure tank. The water intake in the collecting tank is performed through the suction cap with mesh. The water flow rate is controlled by gate valve with electric actuator.

To record the pressure pulses, the test-bench was equipped with a piezoelectric flow rate sensors (type 014MT) designed to convert rapidly varying and pulsed pressure into an electrical signal.
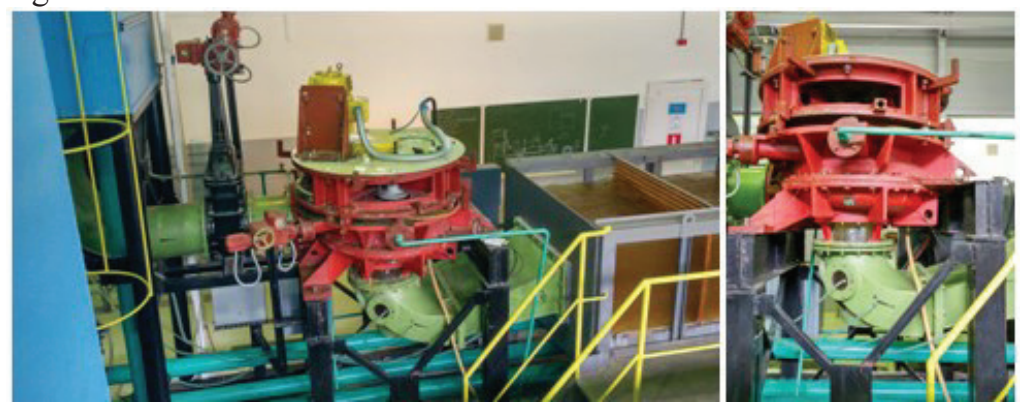

Fig. 1. Model hydro-turbine with radial-axial impeller.

\section{Experimental results}

In the course of experiments we carried out measurements within a wide range of the hydraulic unit operation. The pressure head and the degree of opening of the guide vanes were varied in the experiment. Then all the necessary parameters, namely, impeller rotation frequency, the flow rate through the hydraulic unit, and generating current and voltage were recorded.

The behavior of the frequency and the pressure pulsations intensity in the waterconveyance system of the turbine is of great interest for high-head power plants since the precession of a vortex core is a serious hazard to the hydroturbine equipment. Pressure pulsations were recorded at specified points during five minutes for each of the operation modes.

Figures 2 and 3 below present the results of the experiment carried out for one of the operating modes of the test-bench. The diagrams present time behavior of the pressure pulsation and the pressure pulsation spectrum in the draft tube diffuser. As is obvious, in this mode there is a pronounced frequency of pressure fluctuations associated with the precession of the vortex. 


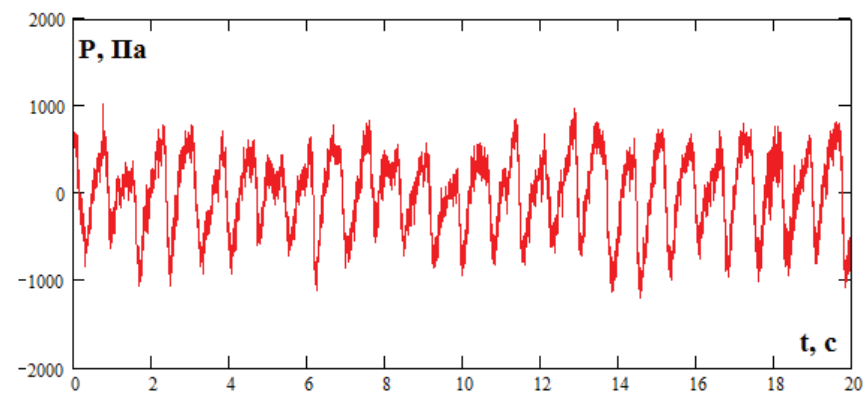

Fig. 2. Time dependence of the pressure pulsation in the draft tube diffuser (opening 3/15; head $H=269 \mathrm{~cm} ; Q=186.5 \mathrm{~m}^{3} / \mathrm{h}$ )

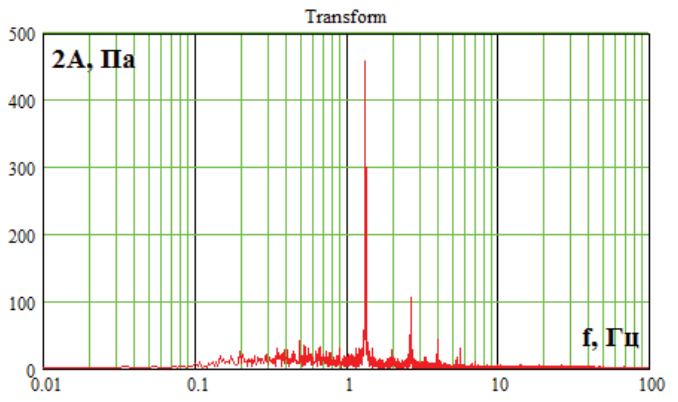

Fig. 3. Spectrum of the pressure pulsations in the draft tube diffuser (opening 3/15; head $\left.H=269 \mathrm{~cm} ; Q=186.5 \mathrm{~m}^{3} / \mathrm{h}\right)$

Figure $4 \mathrm{a}$ shows the pressure pulsation in the draft tube diffuser behind the impeller depending on the opening of the guide vanes. Apparently, the pressure pulsations have a maximum in part-load operation mode at the opening of the guide vanes at $5 \mathrm{~mm}$. Further increase in the opening of the guide vanes and transition of the turbine to the optimal operation mode leads to the decrease in pressure pulsations.

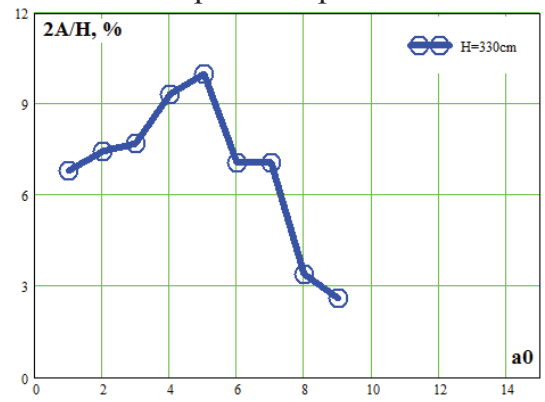

Fig. 4. Dependence of the pressure pulsation amplitude in the draft tube diffuser on the opening of the guide vanes.

Further, to suppress pressure pulsations, air was delivered into the water-conveyance system of the hydraulic turbine. Air was supplied into a scroll chamber of the turbine by two compressors. Air flow rate was monitored by ultrasonic flowmeter.

Figure 6 shows the pressure pulsations spectrum in the draft tube diffuser: (a) corresponds to spectrum without air, while (b) - with air (air flow rate equals $16 \mathrm{~m}^{3} / \mathrm{h}$ ). These results are obtained for the mode of $\mathrm{H}=3 \mathrm{~m}$ and the guide vanes opening at $5 / 15$. This mode was accompanied by the maximum level of pressure pulsations (see Fig. 4). 
The spectra presented in Figs. 6 show that the amplitude of pressure pulsations at the air injection is reduced by almost an order of magnitude. In addition, the fundamental frequency of the pressure pulsations changes upwards. Earlier similar behavior was noted in the works [9] for model test-benches with swirling flow.
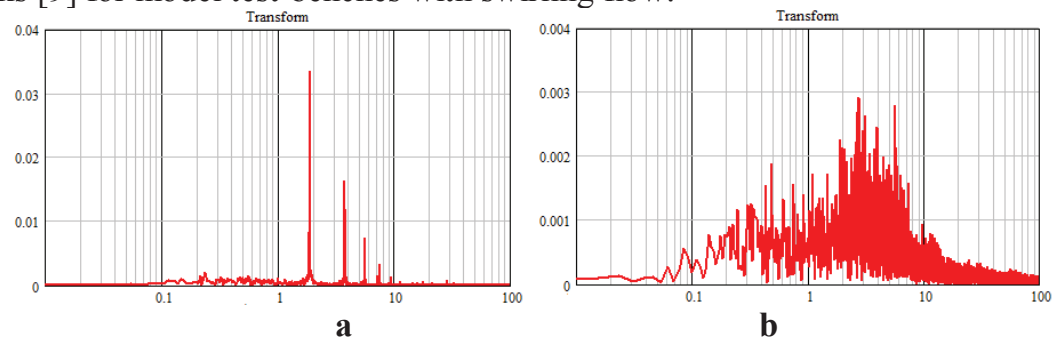

Fig. 6. Pressure pulsations spectrum in the draft tube diffuser; (a) - without air; (b) - with air.

Figure 7 shows the mean-square pressure pulsations at two points located in the draft tube diffuser versus the volumetric flow rate of air. Here the pressure pulsations are reduced to those without air. It is clear that with increasing the flow rate of the intake air, pressure pulsations reduce quite significantly. Thus, at the flow rate of $16 \mathrm{~m}^{3} / \mathrm{h}$, that is approximately $2 \%$ of the water flow rate, pressure pulsations are reduced by about $40 \%$.

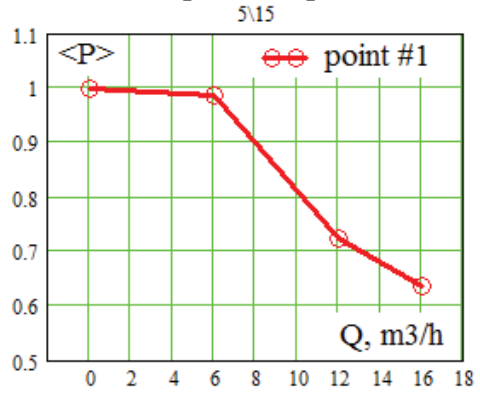

Fig. 7. The root mean square pressure pulsations in the draft tube diffuser depending on the opening of the guide vanes.

\section{Conclusion}

The work deals with experimental investigation of pressure pulsations for different flow regimes in the hydraulic unit of the medium-scale hydrodynamic test-bench. The authors obtained correlations between the pressure pulsations and the opening of the guide vanes for different pressure heads, as well as carried out a preliminary study on the effect of air delivery on pressure pulsations in the hydraulic turbine. The study was performed in the conditions of maximum pressure pulsations. Air was supplied into a spiral chamber of the turbine. This method is one of the most promising to reduce loads on the hydraulic unit, caused by the precession of the vortex core in the space behind the impeller. The results of the study have shown that it is possible to significantly reduce the pressure pulsations using air. However, this effect needs further investigation. In particular, it is necessary to investigate the influence of air on pressure pulsations in other operation modes of the turbine as well as to study the effect of air injection point. 
The current work is performed at partial support of the projects funded by the Russian Foundation for Basic Research and Krasnoyarsk Regional Fund for Support of Scientific and Scientific-Technical Activities (16-48-243076).

\section{References}

1. A.A. Dekterev, A.V. Zakharov, A.V. Minakov, D.V. Platonov, I.M. Pylev, Fluid Dynamics, 50, 5, 601-612

2. A.V. Minakov, A.V. Sentyabov, D.V. Platonov, A.A. Dekterev, A.V. Zakharov, Computer and fluids, 197-205

3. A.V. Minakov, A.V. Sentyabov, D.V. Platonov, A.A. Dekterev, A.V. Zakharov, International Journal of Heat and Fluid Flow, 53, 183-194, (2015)

4. D. Platonov, A. Minakov, D. Dekterev, A. Sentyabov, A. Dekterev, Journal of Physics: Conference Series, 754, (2016)

5. Falvey, H.T., REC-ERC-71-42, U.S. Bureau of Reclamation, 33, (1971)

6. Wahl, T.L., Colorado State University, 77, (1990)

7. Grein, H., Proceedings, $10^{\text {th }}$ Symposium of the International Association for Hydraulic Research Section for Hydraulic Machinery Equipment and Cavitation, Tokyo, Japan, (1980)

8. Bhan, S Proceedings, 5th International Symposium on Hydro Power Fluid Machinery, Chicago, Illinois, 95-102, (1988)

9. Alekseenko S.V., Kuibin P.A., Okulov V.L., Shtork S.I., Heat transfer research, 41, 4, 465-477, (2010) 